\title{
Method of Verifying the Existence of Velocity Vectors Excluded by the Special Theory of Relativity
}

\section{Suto $\mathrm{K}^{*}$}

Independent Researcher, 5-24, Oote-Town, Isesaki, Japan

\begin{abstract}
This paper discusses a thought experiment whose result contradict the predictions of the special theory of relativity. The reason why this contradiction appears is a velocity vector attached to an inertial frame. Since this sort of inertial frame exists in the natural world, the "principle of relativity" that regards all inertial frames as equivalent, cannot be regarded as a true principle. This paper concludes that the special theory of relativity, which assumes the "principle of relativity" in its development, is a theory with a built-in contradiction.
\end{abstract}

Keywords: Special theory of relativity; Velocity vector; Classically stationary frame; Relativistically stationary frame; A priori isotropic propagatiton; Relativistic isotropic propagatiton

\section{Introduction}

According to the "principle of relativity" that was assumed when developing the special theory of relativity (STR), all inertial frames are equivalent. Therefore, the STR denies the existence of inertial frames to which velocity vectors are attached. Einstein developed the STR by asserting that there is no need for the theory to incorporate velocity vectors or the ether [1].

When Einstein developed the STR, he assumed the "principle of relativity" and the "principle of constancy of light speed." The latter includes the following two principles.

"Any ray of light moves in the "stationary" system of co-ordinates with the determined velocity $c$, whether the ray be emitted by a stationary or by a moving body" [2].

"Let a ray of light start at the "A time" $t_{A}$ from A towards B, let it at the "B time" $t_{B}$ be reflected at $\mathrm{B}$ in the direction of $\mathrm{A}$, and arrive again at $\mathrm{A}$ at the "A time" $t_{\mathrm{A}}^{\prime}$.

In agreement with experience we further assume the quantity:

$$
\frac{2 \mathrm{AB}}{t_{\mathrm{A}}^{\prime}-t_{\mathrm{A}}}=c,
$$

to be a universal constant - the velocity of light in empty space" [3].

In this paper, we distinguish between the former principle as the "principle of constancy of light speed I" and the latter principle as the "principle of constancy of light speed II." The "principle of constancy of light speed I" asserts that the light speed in vacuum does not depend on the velocity of the light source. The "principle of constancy of light speed II" asserts that the light speed calculated from the round-trip travel time is constant.

Let there be a given stationary rigid rod of length $L_{0}$ as measured by a ruler which is stationary, and assume that the rod is placed along the stationary frame's $x$-axis.

Assume that clocks A and B of the same type are set up at points $\mathrm{A}$ and $\mathrm{B}$ on the rear (negative direction) and front (positive direction) end of this rod. Here clock A will be abbreviated as $C_{A}$, and clock B as $\mathrm{C}_{\mathrm{B}}$.

Suppose a ray of light is emitted in the direction of B from A at time $t_{A}$ of $\mathrm{C}_{\mathrm{A}}$, reaches and is reflected at $\mathrm{B}$ at time $t_{B}$ of $\mathrm{C}_{\mathrm{B}}$, and then returns to A at time $t_{\mathrm{A}^{\prime}}$ of $\mathrm{C}_{\mathrm{A}}$. Einstein determined that if the following relationships hold between these two times, then the two clocks represent the same time by definition [2].

$$
\begin{aligned}
& t_{\mathrm{B}}-t_{\mathrm{A}}=t_{\mathrm{A}^{\prime}}-t_{\mathrm{B}} \\
& \frac{1}{2}\left(t_{\mathrm{A}}+t_{\mathrm{A}^{\prime}}\right)=t_{\mathrm{B}}
\end{aligned}
$$

Eqns. (1) and (2) can also be applied to an inertial frame $S^{\prime}$ in which a rod is moving at constant velocity relative to a stationary frame $S$. (in this case, $t_{\mathrm{A}}, t_{\mathrm{B}}$ become times in $S^{\prime}$ ).

Now, the rod which was stationary begins to move at constant velocity along the $x$-axis of $S$. At an arbitrary time, a light signal is emitted from point $A$ on the rear side of the rod toward point $B$ on the front side.

If the "principle of constancy of light speed I" is applied, then propagation of light in $S^{\prime}$ (coordinate frame of the rod) seen from an observer in $S$ is anisotropic. Therefore, from the classical perspective, an observer in $S^{\prime}$ determines the propagation of light in $S^{\prime}$ to be anisotropic in the same way. Also, it is concluded that the light speed on the outward path and return path is not $c$.

However, the "principle of constancy of light speed II" also holds in $S$, and thus the light speed calculated by the observer in $S^{\prime}$ from the round-trip time of the light becomes $c$. The STR denies part of this judgment by the observer in $S^{\prime}$ According to the STR, the two inertial frames are equivalent, and thus the light speed measured by the observer in $S^{\prime}$ must be $c$ for both the outward and return path.

Considered classically, an inertial frame in which light propagates isotropically is a "classically stationary frame," and an inertial frame in which light propagates anisotropically is a "classically moving frame."

However, if two clocks in an inertial frame are synchronized using

*Corresponding author: Suto K, Independent Researcher, 5-24, Oote-Town Isesaki, 372-0048, Japan, Tel: 011+81-270-23-9980; E-mail: koshun_suto129@mbr.nifty.com

Received November 03, 2017; Accepted December 24, 2017; Published December 31, 2017

Citation: Suto K (2017) Method of Verifying the Existence of Velocity Vectors Excluded by the Special Theory of Relativity. J Phys Math 8: 258. doi: 10.4172/20900902.1000258

Copyright: (c) 2017 Moffat $\mathrm{J}$, et al. This is an open-access article distributed under the terms of the Creative Commons Attribution License, which permits unrestricted use, distribution, and reproduction in any medium, provided the original author and source are credited. 
the method of Einstein, then even in a "classically moving frame" the light speed is measured as $c$ on both the outward and return path (Relativistic isotropic propagation), just as in a "classically stationary frame."

As a result, both a "classically stationary frame" and a "classically moving frame" fall under the heading of a "relativistically stationary frame," and it is impossible to experimentally identify the two. Also, all inertial frames become stationary frames in the sense of the "principle of relativity."

In this paper, the principle introduced by Einstein is called the "principle of constancy of light speed E" (where "E" stands for Einstein.) That is,

Principle of constancy of light speed E: In all inertial frames, light speed of the outward path and return path is constant $(c)$.

The above points can be summarized as follows:

Classical Physics $\rightarrow$ Special Theory of Relativity

$\left[\begin{array}{l}\text { P rinciple of constancy of lightspeed I } \\ \text { P rinciple of constancy of lightspeed II }\end{array}\right] \rightarrow$ Principle of constancy of lightspeed E

A A priori isotropic propagation

Light signal propagation $\left[\begin{array}{l}\text { A priori isotropic propagation } \\ \text { Anisotropic propagation }\end{array}\right] \rightarrow$ Relativistic isotropic propagation

Inertial frame $\left[\begin{array}{l}\text { Classically stationary frame } S_{\mathrm{cl}} \\ \text { Classically moving frame } S_{\mathrm{cl}}^{\prime}\end{array}\right] \rightarrow$ Relativistically stationary frame $S_{\mathrm{re}}$

Einstein did not treat as a problem the adjustment time of the clocks when synchronizing two clocks. However, in this paper, the adjustment time of clocks is treated as a problem. A method is also presented for identifying $S_{c l}$ and $S_{\mathrm{cl}}^{\prime}$, which Einstein and Michelson regarded as impossible.

\section{Time Adjustment of Clocks Carried out in a Moving Frame}

Without an experiment, it is impossible to conclude that the inertial frame regarded as $S$ in this paper is $S_{\mathrm{cl}}$. Therefore, what can be done at the present time is to assume that that inertial frame is $S_{\mathrm{cl}}$. (Naturally, the STR does not have the concept of $S_{\mathrm{cl}}$. However, later this paper presents a thought experiment enabling identification of $S_{\mathrm{cl}}$ and $S_{\mathrm{cl}}^{\prime}$. For that reason, it is permissible here to assume that the stationary frame is $\left.S_{\mathrm{cl}}\right)$.

Now, consider the case where two rods are placed in a "classically stationary frame" (The two rods will be distinguished as rod I and rod II) (Figure 1).

On rod $\mathrm{I}$, clock $\mathrm{A}$ will be indicated as $\mathrm{C}_{\mathrm{IA}}$ and clock $\mathrm{B}$ will be indicated as $\mathrm{C}_{\mathrm{IB}}$ ( $\mathrm{In}_{\mathrm{IA}}$, I indicates rod $\mathrm{I}$, and $\mathrm{A}$ indicates clock $\mathrm{A}$. The same holds for $\mathrm{C}_{\mathrm{IB}}$ ). The clocks at both ends of rod II will be indicated as $\mathrm{C}_{\mathrm{IIA}}$ and $\mathrm{C}_{\mathrm{IIB}}$.

It is assumed that the times of $\mathrm{C}_{\mathrm{IA}}$ and $\mathrm{C}_{\mathrm{IB}}$, as well as $\mathrm{C}_{\mathrm{IIA}}$ and $\mathrm{C}_{\mathrm{IIB}}$ are synchronized when the clocks are at rest. (Here, the adjustment time is not important. It is sufficient to just synchronize the times of the two clocks).

Once their times have been adjusted, $\mathrm{C}_{\mathrm{IB}}$ will be indicated as $\mathrm{C}_{\mathrm{IB1}}$, and $\mathrm{C}_{\mathrm{IIB}}$ will be indicated as $\mathrm{C}_{\mathrm{IIB1}}$. Here, the times are synchronized when the two clocks are stationary because the author wishes to carry the discussion up to the time adjustment when performing synchronization later.

Also, if the stationary frame is a "classically stationary frame," then

\begin{tabular}{llr}
$\mathrm{C}_{\mathrm{IIA}}$ & $\mathrm{C}_{\mathrm{IB} 1}$ \\
\hline (1) & Rod II & (1) \\
\hline $\mathrm{A}$ & & B \\
\hline (1) & Rod I & C \\
\hline $\mathrm{C}_{\mathrm{IA}}$ & & $\mathrm{C}_{\mathrm{IB} 1}$
\end{tabular}

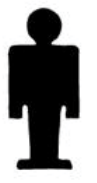

\section{Classically stationary frame}

Figure 1: Two rods with length $L_{0}$ are placed parallel to the $x$-axis of a "classically stationary frame." At this time, the clocks at both ends of the two rods are synchronized. Once their times have been adjusted, $C_{I B}$ will be indicated as $\mathrm{C}_{\mathrm{IB} 1}$, and $\mathrm{C}_{\mathrm{IIB}}$ will be indicated as $\mathrm{C}_{\mathrm{IIB} 1}$.

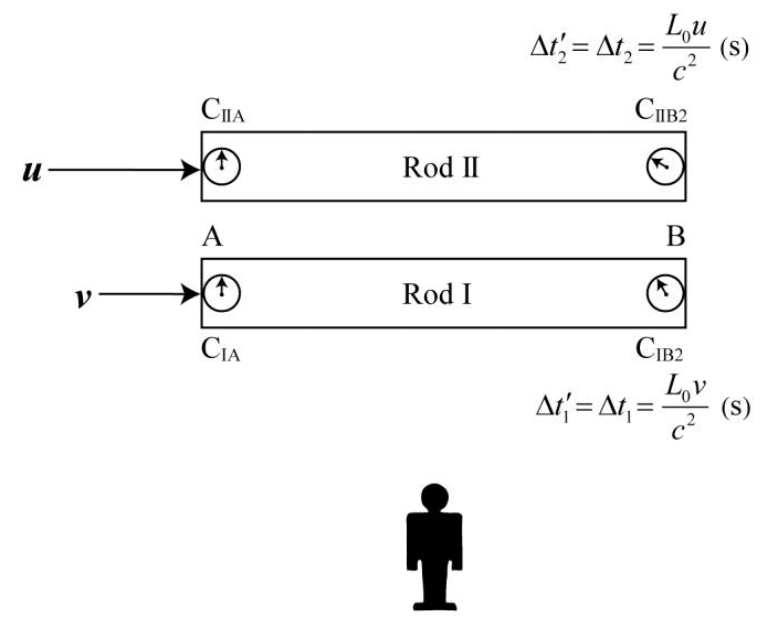

Classically stationary frame

Figure 2: Time adjustment $\Delta t_{1}$ of $C_{1 \mathrm{~B} 1}$ moving at constant velocity $v$ relative to a "classically stationary frame" and time adjustment $\Delta t_{2}$ of $\mathrm{C}_{\mathrm{IB} 1}$ moving in the same way at constant velocity $u$. By making this time adjustment, the coordinate frames of rod I and rod II can maintain their status as "relativistically stationary frames." In this figure, $\Delta t_{1}$ and $\Delta t_{2}$ are times for adjustment by the observer of rod I and rod II, and $\Delta t_{1}^{\prime}$ and $\Delta t_{2}^{\prime}$ are adjustment times predicted by the observer in $S_{c l}$.

$\mathrm{C}_{\mathrm{IA}}$ and $\mathrm{C}_{\mathrm{IB} 1}$, and $\mathrm{C}_{\mathrm{IIA}}$ and $\mathrm{C}_{\mathrm{IIB1}}$, match in an absolute sense by definition.

Next, consider the case when rod I and rod II begin to move at constant velocity, in the positive direction of the $x$-axis of the stationary frame. (Velocity of rod I is assumed to be $v$, and velocity of rod II to be $u$.) It is assumed here that $v<u$ (Figure 2).

Even if the times of the clocks at both ends of a rod match absolutely when at rest, it becomes impossible to say that there is simultaneity from a relativistic perspective when the rod begins to move. Thus, in order to make the times of the two sets of clocks match in the sense of 
the theory of relativity, it is necessary to adjust the time of clock B on two rods. (This can also be done by adjusting clock $\mathrm{A}$, but this paper unifies the treatment in imitation of Einstein by adjusting clock B).

Here, when clock $B$ on rod $I$ is adjusted, the indication $C_{I B 1}$ is changed to $\mathrm{C}_{\mathrm{IB}}$. Also, when clock $\mathrm{B}$ on rod II is adjusted, the indication $\mathrm{C}_{\mathrm{IIB} 1}$ is changed to $\mathrm{C}_{\mathrm{IIB} 2}$. The 2 in ${ }_{\mathrm{B} 2}$ refers to the second time adjustment.

Thought Experiment A: Adjustment time $\Delta t_{1}^{\prime}$ of clock B in the coordinate frame $\left(S_{\mathrm{I}}^{\prime}\right)$ of rod I and adjustment time $\Delta t_{2}^{\prime}$ of clock B in coordinate frame $\left(S_{\mathrm{II}}^{\prime}\right)$ of rod II predicted by an observer in $S_{\mathrm{cl}}$.

Let us measure the time of an event which occurs in another inertial frame by using the clock in $S_{\mathrm{cl}}$. Here, it is assumed that the times $t_{A}, t_{B}, t_{A}$ in $S_{\mathrm{I}}^{\prime}$ correspond to the times $t_{\mathrm{A}}^{\prime}, t_{\mathrm{B}}^{\prime}, S_{\mathrm{I}}^{\prime}$ measured from $S_{\mathrm{cl}}$. (Be careful to note how the prime marks ' are attached).

When the time needed for light emitted from $\mathrm{A}$ in $S_{\mathrm{I}}^{\prime}$ to travel from $\mathrm{A}$ to $\mathrm{B}$ is measured with the clock in stationary frame, the result is $\left(t_{\mathrm{B}}^{\prime}-t_{\mathrm{A}}^{\prime}\right)$.

According to the STR, when viewed from stationary frame, the rod I contracts by $1 / \gamma$ times in the direction of motion [4]. In addition, when the light speed emitted from $S_{\mathrm{I}}^{\prime}$ is seen from stationary frame, it is always constant regardless of the velocity of the light source, and thus $\left(t_{\mathrm{B}}^{\prime}-t_{\mathrm{A}}^{\prime}\right)$ is given by the following equation.

$$
t_{\mathrm{B}}^{\prime}-t_{\mathrm{A}}^{\prime}=\frac{L_{0}}{\gamma(c-v)}(\mathrm{s}), \gamma=\left(1-\frac{v^{2}}{c^{2}}\right)^{-1 / 2}
$$

If the time needed for light to return from $\mathrm{B}$ to $\mathrm{A}$ is measured with the clock in stationary frame, and is taken to be $\left(t_{\mathrm{A}^{\prime}}^{\prime}-t_{\mathrm{B}}^{\prime}\right)$ then:

$$
t_{\mathrm{A}^{\prime}}^{\prime}-t_{\mathrm{B}}^{\prime}=\frac{L_{0}}{\gamma(c+v)}(\mathrm{s})
$$

However, the denominator on the right side of eqns. (3) and (4) does not mean that the light speed varies depend on the velocity of the light source [5].

According to the STR, the relationship between the time $\left(t_{\mathrm{B}}-t_{\mathrm{A}}\right)$ that elapses in $S_{\mathrm{I}}^{\prime}$ and the time $\left(t_{\mathrm{B}}^{\prime}-t_{\mathrm{A}}^{\prime}\right)$ that elapses on a clock in the stationary frame is as follows.

$$
t_{\mathrm{B}}-t_{\mathrm{A}}=\frac{1}{\gamma}\left(t_{\mathrm{B}}^{\prime}-t_{\mathrm{A}}^{\prime}\right)
$$

If the right side of eqn. (3) is substituted for $\left(t_{\mathrm{B}}^{\prime}-t_{\mathrm{A}}^{\prime}\right)$ in eqn. (5),

$$
\begin{aligned}
& t_{\mathrm{B}}-t_{\mathrm{A}}=\frac{L_{0}}{\gamma^{2}(c-v)} \\
& =\frac{L_{0}(c+v)}{c^{2}} \text { (s) }
\end{aligned}
$$

If, in the same way, the time elapsed on a clock in $S_{\mathrm{I}}^{\prime}$ while light returns from $\mathrm{B}$ to $\mathrm{A}\left(t_{\mathrm{A}^{\prime}}-t_{\mathrm{B}}\right)$ is:

$$
t_{\mathrm{A}^{\prime}}-t_{\mathrm{B}}=\frac{L_{0}(c-v)}{c^{2}}
$$

If we set $t_{\mathrm{A}}=0$ to simplify the equation, then the following value is obtained from eqns. (6b) and (7).

$$
\begin{aligned}
& \frac{1}{2} t_{\mathrm{A}^{\prime}}=\frac{1}{2}\left[\left(t_{\mathrm{B}}-t_{\mathrm{A}}\right)+\left(t_{\mathrm{A}^{\prime}}-t_{\mathrm{B}}\right)\right] \\
& =\frac{1}{2}\left[\frac{L_{0}(c+v)}{c^{2}}+\frac{L_{0}(c-v)}{c^{2}}\right] \\
& =\frac{L_{0}}{c}
\end{aligned}
$$

When light travels from A to B in $S_{1}^{\prime}$ an observer in stationary frame predicts that $L_{0}(c+v) / c^{2}$ (s) have passed on the clock in $S_{I}^{\prime}$ However, when this light which left $\mathrm{A}$ at $t_{\mathrm{A}}=0$ reaches $\mathrm{B}$, by definition, the time shown on clock B must be $L_{0} / c(s)$.

However, since $L_{0}(c+v) / c^{2}>L_{0} / c$, the time on clock B must be later than the time on clock A to resolve this discrepancy. Thus, if the time adjustment to actually make the time on clock B later is $\Delta t_{1}$, it should be possible to take the difference between the two as this time. Namely,

$$
\begin{aligned}
& \Delta t_{1}=\left(t_{\mathrm{B}}-t_{\mathrm{A}}\right)-\frac{1}{2} t_{\mathrm{A}^{\prime}} \\
& =\frac{L_{0}(c+v)}{c^{2}}-\frac{L_{0}}{c} \\
& =\frac{L_{0} v}{c^{2}} \text { (s) }
\end{aligned}
$$

$\Delta t_{1}$ matches with the adjustment time $\Delta t_{1}^{\prime}$ predicted by an observer in the stationary frame. If an observer in $S_{\mathrm{I}}^{\prime}$ delays the time on clock B by $L_{0} v / c^{2}(\mathrm{~s})$, then the relationship in eqn. (1) will hold in this coordinate frame.

Now we synchronize the time of clocks on both ends of rod II. If the same method is used as when the time of $\mathrm{C}_{\mathrm{IB1} 1}$ on rod I was adjusted, then in this case too, the adjustment time $\Delta t_{2}^{\prime}$ of $\mathrm{C}_{\text {IIB1 }}$ predicted by an observer in the stationary frame matches with the adjustment time $\Delta t_{2}$ actually performed by observer II, and the result is as follows.

$$
\Delta t_{2}^{\prime}=\Delta t_{2}=\frac{L_{0} u}{c^{2}}(\mathrm{~s})
$$

In eqn. (10), $v$ in eqn. (9c) is replaced with $u$.

Thought Experiment B: Adjustment time $\Delta t_{3}^{\prime}$ of clock B on rod II predicted by observer I of rod I, and time $\Delta t_{3}$ actually adjusted by the observer II of rod II.

Next, assume that rod II does not move from the beginning at constant velocity $\boldsymbol{u}$, and instead that it originally moves at the same constant velocity $\boldsymbol{v}$ as rod I (Figure 3).

Incidentally, in order for observer I to predict the adjustment time
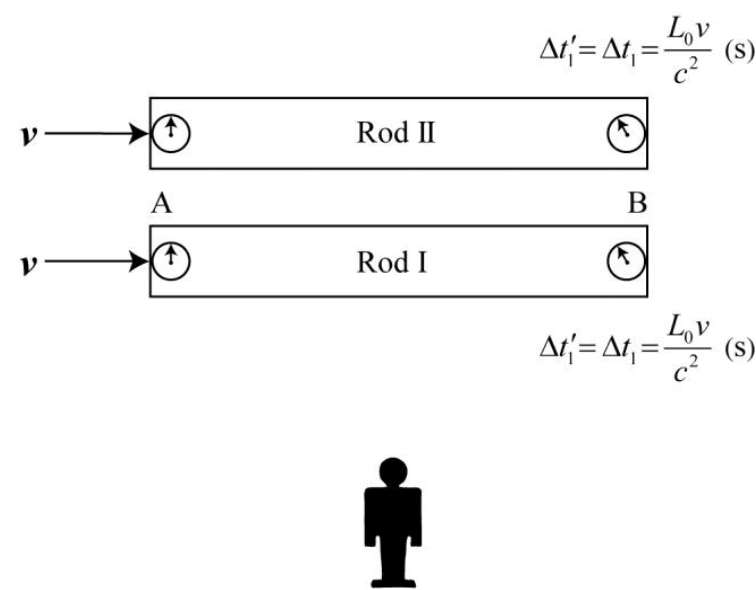

Classically stationary frame

Figure 3: In this case, at the stage before rod II attains the constant velocity $u$, it moves at constant velocity $v$. The time of clock B on rod II is adjusted at that time. 
of clock B of rod II, it is necessary to synchronize clocks A and B on rod II while the two rods are translational motion as in Figure 3.

The velocity addition law in STR is given by the following equation.

$$
u=\frac{v+w}{1+\frac{v w}{c^{2}}}
$$

This indicates that when the relative velocity of the stationary frame and rod II is $\boldsymbol{u}$, the relative velocity of rod I and $\operatorname{rod}$ II is $w$.

Now, observer I predicts the adjustment time of clock B of rod II moving at constant velocity $w$ relative to rod I (Figure 4 ).

The observer of rod I applying the "principle of relativity" regards his own coordinate frame as a stationary frame. Thus, using the same logic as in Thought Experiment A, this observer predicts that the adjustment time $\Delta t_{3}^{\prime}$ of clock B on rod II will be as follows.

$$
\Delta t_{3}^{\prime}=\frac{L_{0} w}{c^{2}}(\mathrm{~s})
$$

However, to simply predict $\Delta t_{3}^{\prime}$ there is no need for the first time adjustment of the two clocks B in Figure 1. The time adjustment of the two clocks $B$ can be done when the rod has started moving at constant velocity. (However, in this case the time adjustment cannot be predicted.)

Next, the adjustment time of clock B on rod II, which has attained the constant velocity $\boldsymbol{u}$, is predicted from the stationary frame $S_{\mathrm{cl}}$.

If here the adjustment time of clock $\mathrm{B}$, predicted by the observer in $S_{\mathrm{cl}}$ is assumed to be $\Delta t_{4}^{\prime}$

$\Delta t_{4}^{\prime}=\Delta t_{2}^{\prime}-\Delta t_{1}^{\prime}$

$\Delta t_{4}^{\prime}$ is as follows, due to eqns. (10) and (9c).

$\Delta t_{4}^{\prime}=\frac{L_{0}(u-v)}{c^{2}}$

Therefore, the adjustment time $\Delta t_{3}$ actually carried out by the observer of rod II is as follows.

$$
\Delta t_{3}=\Delta t_{2}-\Delta t_{1}=\Delta t_{2}^{\prime}-\Delta t_{1}^{\prime}=\Delta t_{4}^{\prime}
$$

$$
\Delta t_{3} \neq \frac{L_{0} w}{c^{2}}(\mathrm{~s})
$$

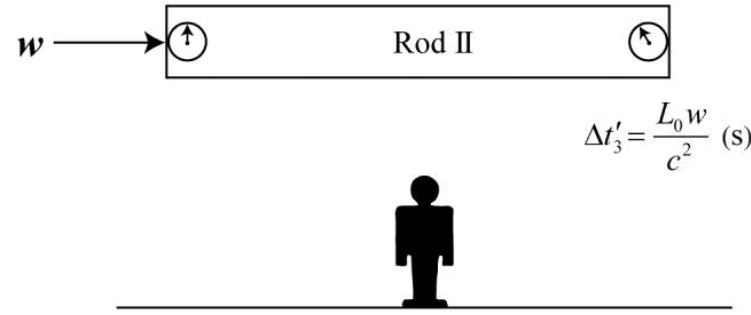

Observer on Rod I

\section{Classically moving frame}

Figure 4: The case when rod II in Figure 2 is viewed by the observer of rod I. In this case, the observer of rod I believes that his own coordinate frame is a stationary frame, and thus he believes that the time adjustment $\Delta t_{3}^{\prime}$ of $\mathrm{C}$ is $L_{0} w / c^{2}(\mathrm{~s})$. This time does not match the time $\Delta t$ of adjustment by the observer of rod II. A velocity vector is attached to the coordinate frame of rod I, which is regarded as a stationary frame from the standpoin of the theory of relativity. Since this sort of inertial frame exists in the natural world, the "principle of relativity" that regards all inertial frames as equivalent, cannot be regarded as a true principle.
Also,

$$
\Delta t_{3}^{\prime} \neq \Delta t_{3}
$$

The cause of the mismatch between $\Delta t_{3}$ and $\Delta t_{3}^{\prime}$ is the velocity vector attached to the coordinate frame of rod I (Figure 4) regarded as a stationary frame from the perspective of the STR (Appendix).

Next, let us find the size of the component in the $x$-axis direction of the unknown velocity vector $\boldsymbol{v}_{x}$ which causes this mismatch of $\Delta t_{3}$ and $\Delta t_{3}^{\prime}$ Assume here that $\Delta t_{3} / \Delta t_{3}^{\prime}$ is a. That is,

$$
\frac{\Delta t_{3}}{\Delta t_{3}^{\prime}}=\frac{u-v_{x}}{w}=\mathrm{a}, \quad 0<\mathrm{a}<1
$$

If the $u$ in eqn. (17) is eliminated by using eqn. (11), then an is as follows:

$$
\mathrm{a}=\frac{c^{2}-v_{x}^{2}}{c^{2}+v_{x} w}
$$

From this, it is possible to derive a quadratic equation like the following for $v_{x}$ :

$$
v_{x}^{2}+\mathrm{a} w v_{x}-(1-\mathrm{a}) c^{2}=0
$$

If this equation is solved while taking into account that the size of $\boldsymbol{v}_{x}$ is positive, then $\boldsymbol{v}_{x}$ is as follows:

$$
v_{x}=\frac{-\mathrm{a} w+\left[\mathrm{a}^{2} w^{2}+4(1-\mathrm{a}) c^{2}\right]^{1 / 2}}{2}
$$

\section{Conclusion}

In the STR, the adjustment time, when synchronizing two clocks placed in a certain inertial frame, was not treated as a problem. However, in this paper, the adjustment time of clocks is treated as a problem.

In Figure 1, rod I is stationary in $S_{\mathrm{cl}}$ (Naturally, rod II is also stationary.) Also, in Figure 3, rod II is stationary relative to the coordinate frame of rod I.

We considered the situation where a rod is moving at constant velocity relative to these two types of stationary frames. (rod I in Figure 2 and rod II in Figure 4.)

A. If the adjustment time of clock B of rod I predicted by the observer in $S_{\mathrm{cl}}$ in Figure 2 is taken to be $\Delta t_{1}^{\prime}$, and the time of actual adjustment by the observer of rod I is taken to be $\Delta t$ then:

$$
\Delta t_{1}^{\prime}=\Delta t_{1}=\frac{L_{0} v}{c^{2}}
$$

When eqn. (21) holds, light propagates isotropically in the a priori sense in the stationary frame of Figure 2 where the rod was stationary at the beginning. It can be concluded that this stationary frame is $S_{\mathrm{cl}}$.

B. In Figure 4, rod II is moving at constant velocity $w$ relative to the coordinate frame of rod I. At this time, the adjustment time of clock B predicted by the observer of rod I is taken to be $\Delta t_{3}^{\prime}$, and the time of actual adjustment by the observer of rod II is taken to be $\Delta t_{3}$.Also, if the adjustment time of clock B predicted by the observer in $S_{\mathrm{cl}}$ is taken to be $\Delta t_{4}^{\prime}$, then:

$$
\begin{aligned}
& \Delta t_{3}^{\prime} \neq \Delta t_{3} \\
& \Delta t_{4}^{\prime}=\Delta t_{3}=\frac{L_{0} w}{c^{2}}\left(\frac{c^{2}-v_{x}^{2}}{c^{2}+v_{x} w}\right)(\mathrm{s})
\end{aligned}
$$

When eqn. (22) holds, light propagates anisotropically in the 
Citation: Suto K (2017) Method of Verifying the Existence of Velocity Vectors Excluded by the Special Theory of Relativity. J Phys Math 8: 258. doi: 10.4172/2090-0902.1000258

Page 5 of 5

coordinate frame of rod I in Figure 4. It can be concluded that the coordinate frame of this rod I is $S_{\mathrm{cl}}^{\prime}$.

This paper has shown, using a thought experiment for determining the existence of a velocity vector attached to an inertial frame, that it is possible to identify $S_{\mathrm{cl}}$ and $S_{\mathrm{cl}}^{\prime}$. The paper has clarified that the "principle of relativity," whereby all inertial frames are taken to be equivalent, is not truly a principle [4,6-9]. The paper concludes that the STR, which assumes the "principle of relativity" in its development, is a theory with a built-in contradiction.

\section{References}

1. Einstein A (1923) The Principle of Relativity. New York, Dover, USA, p: 38

2. Einstein A (1923) The Principle of Relativity. New York, Dover, USA, p: 41
3. Einstein A (1923) The Principle of Relativity. New York, Dover, USA, p: 40.

4. Suto K (2017) An Elucidation of the Symmetry of Length Contraction Predicted by the Special Theory of Relativity. Applied Phys Research 9: 31.

5. Suto K (2010) Violation of the special theory of relativity as proven by synchronization of clocks. Phys Essays 23: 511.

6. Suto K (2016) Thought Experiment Revealing a Contradiction in the Special Theory of Relativity. Applied Phys Research 8: 70.

7. Suto K (2016) Elucidation of Time Symmetry Predicted by the Special Theory of Relativity. IOSR Journal of Applied Physics 8: 20.

8. Suto K (2017) A New Problem with the Twin Paradox. Applied Phys Research 9: 77.

9. Suto K (2015) Demonstration of the existence of a velocity vector missing from the special theory of relativity. Phys Essays 28: 345 